\title{
EPITHELIOID TROPHOBLASTIC TUMOUR: A RARE CASE WITH AN UNUSUAL PRESENTATION
}

\author{
Jayanandhini Manoharan1, Kanchana Madurai Padmanaban² \\ 1 Post Graduate, Department of Pathology, Institute of Obstetrics and Gynaecology, Madras Medical College, Chennai. \\ 2 Professor, Department of Pathology, Institute of Obstetrics and Gynaecology, Madras Medical College, Chennai.
}

\begin{abstract}
Here in we report a rare case of epithelioid trophoblastic tumour with a rare clinical presentation. Commonly, ETT presents with abnormal vaginal bleeding or lung metastases. Our case was a 22-year-old lady, Para 2, Live 2, Last Child Birth was one year with History of amenorrhoea presented with abdominal pain on and off, and initial Ultrasonogram had fluid in Pouch of Douglas with elevated beta HCG levels- $421.4 \mathrm{miu} / \mathrm{mL}$. She was diagnosed as ectopic pregnancy. Laparotomy was done, which turned out to be negative. So patient was referred to our institute with abdominal distension and ascites. MRI showed heterogeneously enhancing lesion in myometrium, lateral wall of cervix and anterior wall of vagina with gross ascites. Total abdominal hysterectomy with bilateral salpingo-oophorectomy was done. Microscopy showed a neoplasm composed of round to polygonal intermediate trophoblastic cell arranged in sheets and clusters, the cells with high-grade nucleus and mitotic activity. Tumour cells were seen infiltrating the myometrium with extensive areas of necroses. Immunohistochemistry showed P63 strong positive, inhibin +ve, EMA +VE, PLAP +VE, beta HCG and Desmin were negative. Histology and IHC were consistent with Epithelioid trophoblastic tumour. This case shows that ETT can also present as abdominal pain with elevated beta HCG and mimic an ectopic pregnancy. The case is presented for its challenging diagnostic dilemma and rare presentation as abdominal distension and ascites.
\end{abstract}

\section{KEYWORDS}

Epithelioid Trophoblastic Tumour, Ectopic, Inhibin, EMA.

HOW TO CITE THIS ARTICLE: Manoharan J, Padmanaban KM. Epithelioid trophoblastic tumour: a rare case with an unusual presentation. J. Evolution Med. Dent. Sci. 2016;5(57):3970-3972, DOI: 10.14260/jemds/2016/908

\section{INTRODUCTION}

Gestational trophoblastic diseases are a group of disorders composed of benign lesions like complete mole, partial mole, invasive mole, exaggerated placental site reaction, placental site nodule and malignant lesions like choriocarcinoma, placental site trophoblastic tumour, epithelioid trophoblastic tumour.(1) ETT is a very rare tumour, first described by Shih and Kurman in 1998, and so far only 94 cases are reported worldwide.(2) Herein we present a case with abdominal pain and elevated HCG levels, which was diagnosed as ruptured ectopic pregnancy. Hence proceeded with laparotomy, which turned out to be negative and was referred to our institute with abdominal distension and ascites and subsequently diagnosed as Epithelioid trophoblastic tumour.

\section{CASE HISTORY}

Our case was a 22-year-old lady, Para 2, Live 2 with Last Child Birth one year back. She presented with a history of amenorrhoea and abdominal pain on and off. Ultrasonogram showed fluid in Pouch of Douglas. Serum beta HCG levels were elevated to $-421.4 \mathrm{miu} / \mathrm{mL}$. As uterus was empty, she was diagnosed as ruptured ectopic pregnancy and was proceeded with a laparotomy in a private hospital. Since laparotomy turned out to be negative, patient was referred to our institute for evaluation with abdominal distension and ascites and elevated beta HCG levels. MRI showed heterogeneously enhancing lesion in myometrium, lateral wall of cervix and anterior wall of vagina with gross ascites.

Financial or Other, Competing Interest: None.

Submission 09-06-2016, Peer Review 03-07-2016,

Acceptance 09-07-2016, Published 18-07-2016.

Corresponding Author:

Dr. Jayanandhini Manoharan

No. 4, Patel Street, Nehru Nagar,

Chrompet,

Chennai-600044.

E-mail: jayanandhiniram@gmail.com

DOI: $10.14260 /$ jemds/2016/908
Hence proceeded with total abdominal hysterectomy and bilateral salpingo-oophorectomy.

\section{Gross}

We received uterus with cervix measuring $11 \times 5 \times 3 \mathrm{~cm} . \mathrm{C} / \mathrm{S}$ showed irregular ulcerated area in the lower uterine segment with endometrium showing tiny polypoid excrescences and a friable polypoid growth extending into endocervix.

\section{Microscopy}

Showed a neoplasm composed of round to polygonal intermediate trophoblastic cells arranged in sheets and clusters. The tumour cells exhibit moderate to abundant cytoplasm with high-grade nucleus and prominent nucleoli with mitotic activity 5-10/HPF. Tumour cells were seen infiltrating into myometrium. Areas of necroses seen. Nonneoplastic endometrium was not seen. Ectocervix appeared normal in histology.

\section{Immunohistochemistry Showed}

- $\quad$ P63 Strong positive.

- Inhibin positive.

- EMA positive.

- $\quad$ PLAP positive.

- $\quad$ Beta HCG and Desmin were negative.

- Histology and IHC were consistent with Epithelioid Trophoblastic Tumour.

- $\quad$ Ki 67 positive

\section{DISCUSSION}

Three types of trophoblastic cells are seen in the placental pathology. They are syncytiotrophoblast, cytotrophoblast and intermediate trophoblasts. Intermediate trophoblasts are of two types, implantation type and chorionic type. Tumour of implantation type intermediate trophoblast is placental site trophoblastic tumour and tumour of chorionic type 
intermediate trophoblast is Epithelioid Trophoblastic Tumour (ETT).

Maximum number of cases are reported in the reproductive age group. Most common presentation is abnormal vaginal bleeding and lung metastases. $(3,4)$ Our case is the 3rd case in literature to the best of our knowledge to present with abdominal pain followed by abdominal distension and ascites. According to Noh et al 2008, only one case was reported.(5) Most common site is uterus - $44 \%$ followed by cervix and most common extrauterine site was lung. $(1,4,6,7,8)$ According to Palmer et al, ETT presents with elevated beta HCG levels, but usually less than 2500 .

Histologically, ETT can be differentiated from Placental Site Trophoblastic Tumour (PSTT), by the cells showing marked pleomorphism and vascular invasion in PSTT. ETT is distinguished from choriocarcinoma by dimorphic population of cells in them.

Immunohistochemically, ETT shows diffuse positivity for inhibin and epithelial membrane antigen. Focal positivity for PLAP and HPL and beta HCG.(9)

P63 is found in chorionic type IT and not in implantation type. Hence, it helps in differentiating ETT from PSTT.(10,11) Ki67 labelling index is useful to distinguish ETT (>12\%) from placental site nodule $(<8 \%)$.

History, clinical features, histology and IHC are valuable tools for properly categorising the trophoblastic lesions. As the management protocol differs for each of them, accurate diagnoses of ETT followed by surgical management gives good prognoses. Our patient is doing well and beta HCG has touched baseline and is under followup.

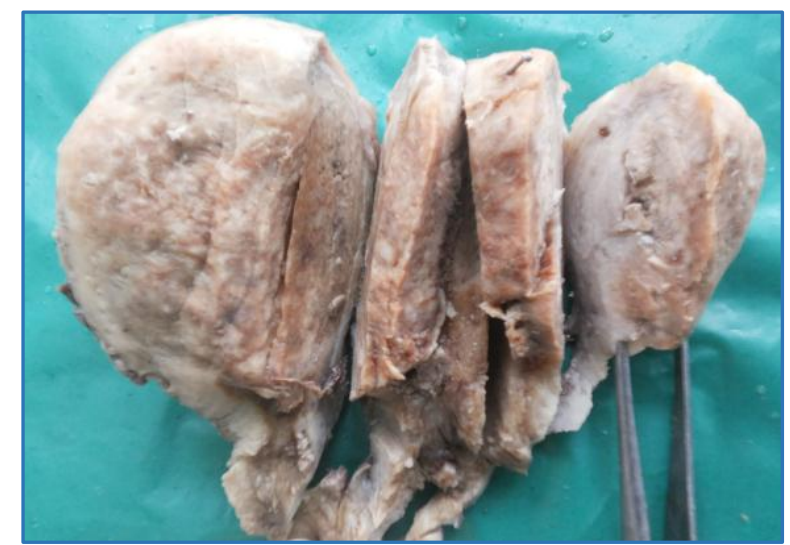

Fig. 1: Showing Growth in the Lower Uterine Segment and Cervix with Distorted Uterine Cavity and Cervical Canal

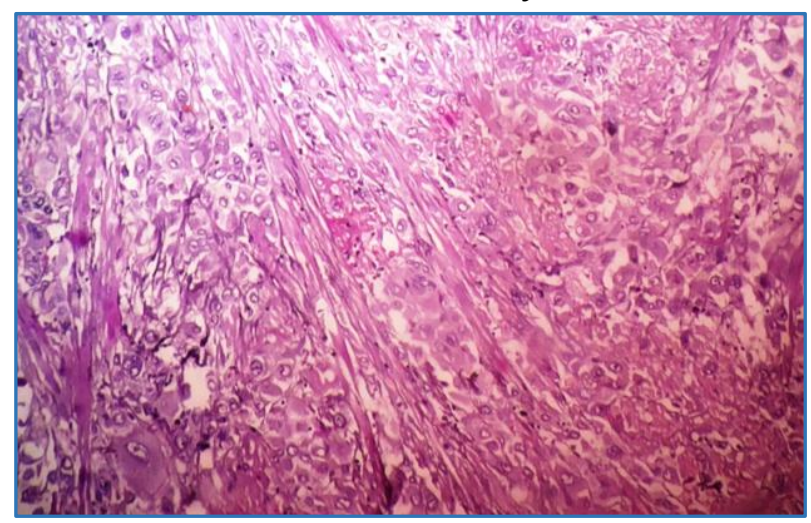

Fig. 2: H\&E 100X Sheets and Clusters of Pleomorphic Trophoblasts Round to Polygonal Infiltrating the Myometrium

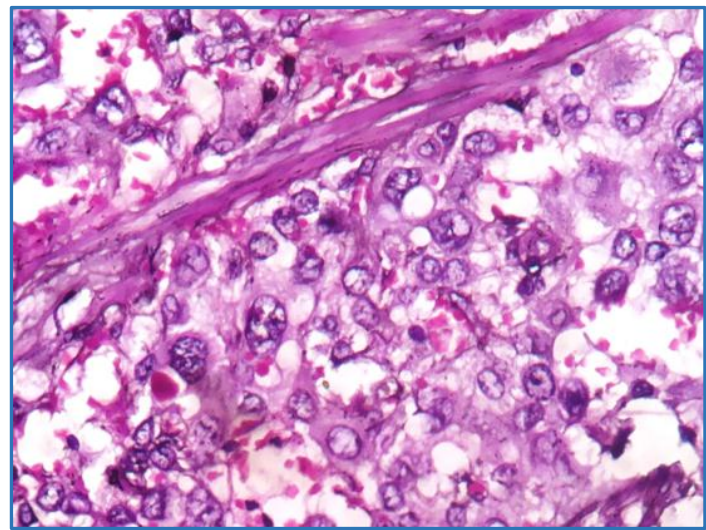

Fig. 3: H\&E Intermediate Trophoblasts with Abundant Cytoplasm, Nuclear Pleomorphism, Prominent Nucleoli are seen with Areas of Hyalinising Necroses

\section{IMMUNOHISTOCHEMISTRY}

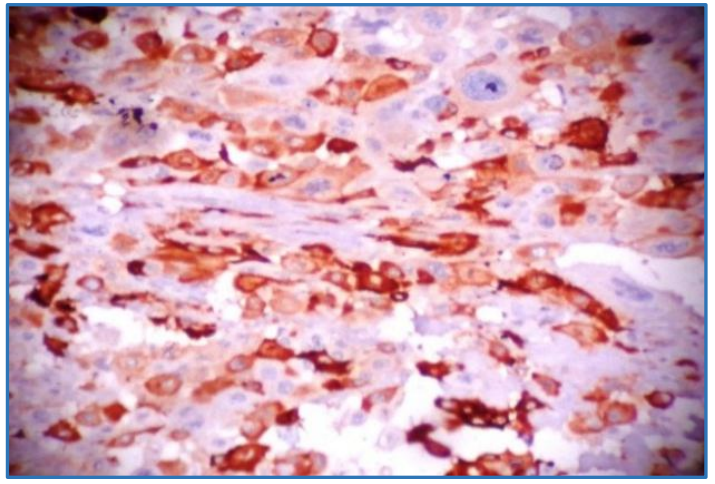

Fig. 4: EMA-Strong Cytoplasmic Positivity

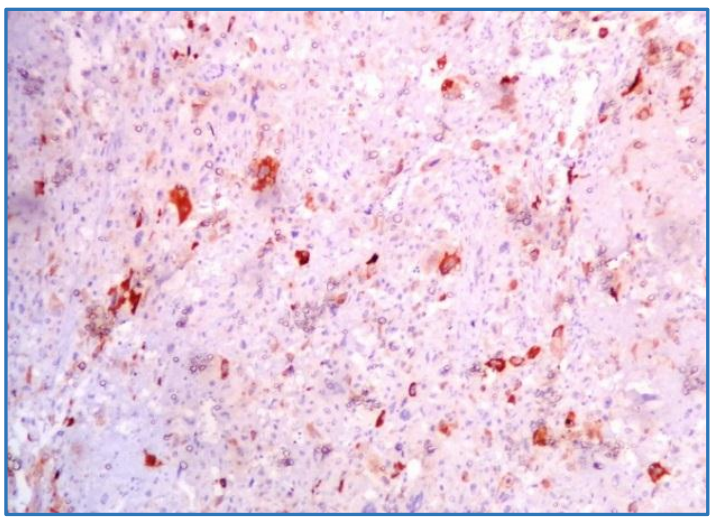

Fig. 5: Inhibin-Positive

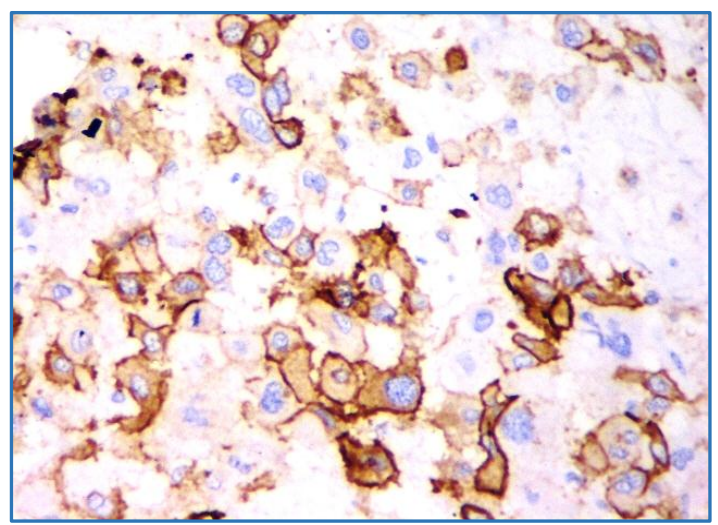

Fig. 6: PLAP - Positive 


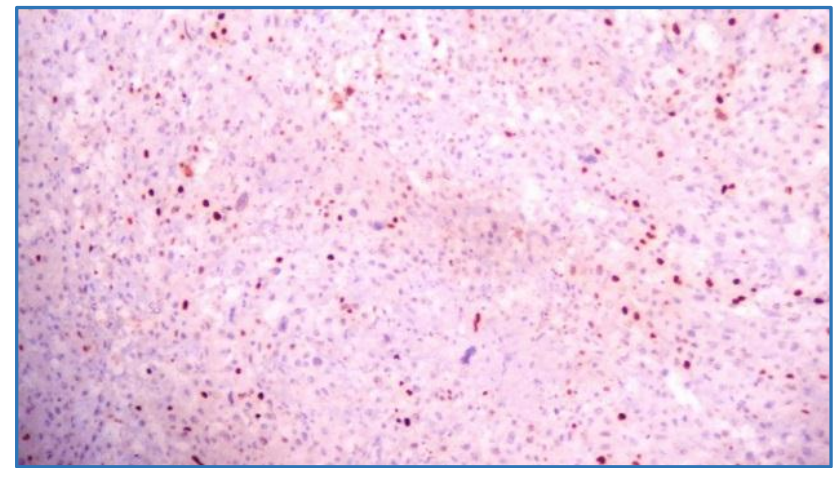

Fig. 7: P63 Positive

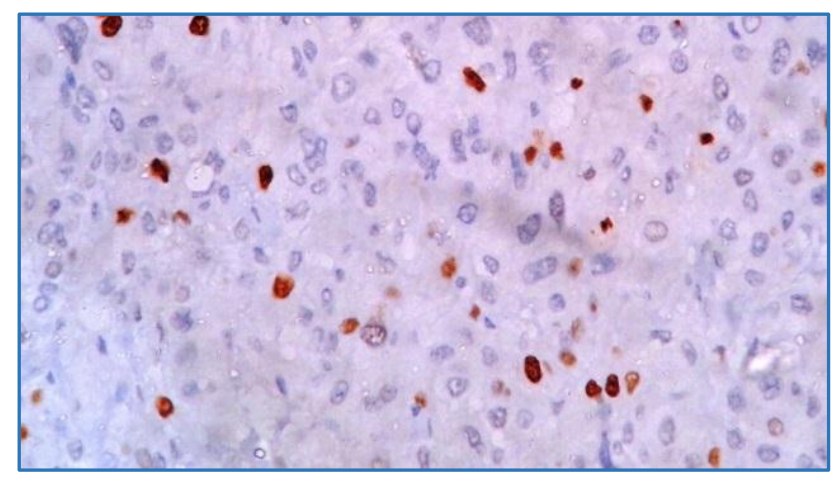

Fig. 8: Ki-67 Positive

\section{CONCLUSION}

ETT is an aggressive trophoblastic lesion amenable to surgery and not to chemotherapy, unlike other gestational trophoblastic lesions. It also has variegated presentation as in our case as abdominal pain with elevated beta HCG, mimicking an ectopic pregnancy. Subsequently presented with abdominal distension and ascites, which is another uncommon presentation. Prompt knowledge of these unusual presentations is needed for accurate diagnosis. The case is presented for its challenging diagnostic dilemma due to its variegated presentations.

\section{ABBREVIATIONS}

ETT - Epithelioid Trophoblastic Tumour.

\section{REFERENCES}

1. Kurman RJ, Ellenson LH, Ronnett BM. Blausteins pathology of the female genital tract. $6^{\text {th }}$ edn. Springer Sciencep Business Media. Chapter 20, New York, Dodrecht Heidelberg London, 2011:pgs 1075.
2. Coulson LE, Kong CS, Zaloudek C. Epithelioid trophoblastic tumor of the uterus in a postmenopausal woman: a case report and review of the literature. The American Journal of Surgical Pathology 2000;24(11): 1558-62.

3. Shih IM, Kurman RJ. Epithelioid trophoblastic tumor: a neoplasm distinct from choriocarcinoma and placental site trophoblastic tumor simulating carcinoma. The American Journal of Surgical Pathology 1998;22(11): 1393-403.

4. Palmer JE, Macdonald M, Wells BW, et al. Epithelioid trophoblastic tumor: a review of the literature. Journal of Reproductive Medicine for the Obstetrician and Gynecologist 2008;53(7):465-75.

5. Noh HT, Lee KH, Lee MA, et al. Epithelioid trophoblastic tumor of paracervix and parametrium. International Journal of Gynecology \& Cancer 2008;18(4):843-6.

6. Vencken PM, Ewing PC, Zweemer RP. Epithelioid trophoblastic tumor: a case report and review of literature. J Clin Pathol 2006;59(12):1307-08.

7. Lewin SN, Aghajanian C, Moreira AL, et al. Extrauterine epithelioid trophoblastic tumors presenting as primary lung carcinomas: morphologic and immunohistochemical features to resolve a diagnostic dilemma. The American Journal of Surgical Pathology 2009;33(12):1809-14.

8. Urabe S, Fujiwara H, Miyoshi H, et al. Epithelioid trophoblastic tumor of the lung. Journal of Obstetrics and Gynaecology Research 2007;33(3):397-401.

9. Shih IM. Trophogram an immunohistochemistry-based algorithmic approach, in the differential diagnosis of trophoblastic tumors and tumor-like lesions. Annals of Diagnostic Pathology 2007;11(3):228-34.

10. Shih IM, Kurman RJ. P63 expression is useful in the distinction of epithelioid trophoblastic and placental site trophoblastic tumors by profiling trophoblastic subpopulations. The American Journal of Surgical Pathology 2004;28(9):1177-83.

11. Kalhor N, Ramirez PT, Deavers MT, et al. Immunohistochemical studies of trophoblastic tumors. The American Journal of Surgical Pathology 2009;33(4): 633-8. 